\title{
Experimental study on the effect of chitosan biopolymer on sandy soil stabilization
}

\author{
Nader Shariatmadari ${ }^{1, *}$, Mohammad Reza ${ }^{1}$, Amiri Tasuji ${ }^{1}$, Pooria Ghadir $^{1}$, and A. Akbar Javadi ${ }^{2}$ \\ ${ }^{1}$ Department of Civil Engineering, Iran University of Science and Technology, Tehran, Iran. \\ ${ }^{2}$ Department of Engineering, University of Exeter, Exeter, United Kingdom.
}

\begin{abstract}
Due to the environmental impacts of conventional soil stabilization materials, such as cement, ongoing efforts have been carried out by different researchers to find alternative economical materials for substitution. Biopolymers are environmentally friendly materials that are widely used in different geoenvironmental applications such as removal of heavy metals from contaminated soils, reduction of soil hydraulic conductivity, erosion control, and soil improvement. In this research the feasibility of using chitosan biopolymer for sandy soil stabilization has been studied. The effects of biopolymer content, curing time, and curing conditions have investigated using unconfined compression tests. The results indicated that incorporation of chitosan has the potential to increase the interparticle cohesion between the particles and considerable improvement of sandy soil mechanical properties. After initial strengthening of the soil, some strength reduction over time was observed due to the degradation characteristics of the chitosan. With regards to the curing condition, better performances at dry condition compare to the wet and saturated environment were achieved. In addition to soil mechanical properties, the pore plugging effect of chitosan biopolymer on highly permeable sandy soil has been studied in this study.
\end{abstract}

\section{Introduction}

Increases in greenhouse gases such as carbon dioxide $\left(\mathrm{CO}_{2}\right)$ have harmful effects on the environment including global warming. Global warming can cause earthquakes, tsunamis, and volcanoes [1]. One of the reasons for the increase in $\mathrm{CO}_{2}$ in space is cement production. Cement production produces about 6 percent of all carbon dioxide released into space [2]. Cement is one of the most common and widely used materials in construction and engineering works. Cement is also widely used to improve soil mechanical properties and soil stabilization in geotechnical engineering. Therefore, to improve the mechanical properties of soils, novel materials are needed that will be less harmful to the environment. A new and environmentally friendly way to improve the mechanical and hydraulic properties of soil is the use of biopolymers. Biopolymers are produced from renewable natural sources such as gum trees, shrimp shell, milk, fermentation of glucose, algae, fungus or bacteria that consist of polysaccharides and reduce $\mathrm{CO}_{2}$ emissions continuously [3-5]. There have been numerous studies on the use of biopolymers in food production, agriculture, cosmetics, medicine, pharmaceuticals and engineering [6, 7]. Recently, researchers have been investigating the use of biopolymers in the field of geotechnical engineering $[3,8]$. Shear strength parameters (cohesion and friction angle) of biopolymer stabilized soil have been studied in recent researches [9]. In general, four factors influence the increase of soil strength by biopolymers which are: type of soil, hydration level (e.g., moisture content), xanthan gum content, and mixing method [8, 10, 11]. While, thermal treatment and air-drying process are main factors in soil strength enhancement by thermo-gelation biopolymers like gellan gum and guar gum $[12,13]$. Furthermore, the role of environmental condition on longterm performance of biopolymer stabilized soil is significant. Cyclic wetting and drying of gellan gum-treated sands show that wetting reduces the strength of the improved soil significantly and after 10 times wetting and drying, the improved dried sample regains $70 \%$ initial strength [14].

Chitosan is a biopolymer usually obtained from the deacetylation of chitin [15]. Chitosan is synthesized from various sources such as shrimp, crab, krill, Crawfish and lobster. It is estimated that shell waste from shrimp and crab harvest is estimated at about 1.44 million tons annually [16]. There are various methods for chitosan synthesis. The properties of chitosan and its applications depend directly on the degree of acetylation and molecular weight of chitosan [15]. In environmental applications, chitosan is used for removal of water contamination such as heavy metals, hydro carbon and herbicides [17-19].

In geotechnical contexts, chitosan has been used as an effective material to prevent soil and water erosion $[20,21]$. Water drop test showed that low concentration chitosan solutions can be used as a coating or mixed with materials to protect against water drop erosion [22].

*Corresponding author: shariatmadari@iust.ac.ir 
Most gel-type biopolymers show minimal interaction with cohesionless sand, due to the neutral surface of the sand particles. On the other hand, although the influence of chitosan incorporation on mechanical and microstructure behavior of fine grained soil has been evaluated [3], chitosan biopolymer interaction with neutral sand particles has not been investigated comprehensively. Therefore, in this study, chitosan biopolymer was used to improve the mechanical properties and hydraulic conductivity of sandy soil. The differences between the mechanism of this bio-material in clayey and sandy soil mechanical improvement are discussed clearly.

\section{Materials and methods}

\subsection{Soil characteristics}

The sandy soil in this study was selected from Firoozkuh-Iran. In order to identify the physical properties of this soil, grain size distribution, maximum and minimum soil porosity $\left(\mathrm{e}_{\max }\right.$ and $\left.\mathrm{e}_{\min }\right)$, and specific gravity of soil (Gs) tests were performed, the results of which are presented in Table 1.

Table 1. Soil characteristics of Firoozkuh sand.

\begin{tabular}{|c|c|c|c|c|c|}
\hline$G \boldsymbol{s}$ & $\boldsymbol{e}_{\max }$ & $\boldsymbol{e}_{\min }$ & $\boldsymbol{D}_{50}(\mathbf{m m})$ & $\boldsymbol{C}_{\boldsymbol{u}}$ & $\boldsymbol{C}_{\boldsymbol{c}}$ \\
\hline 2.658 & 0.943 & 0.603 & 0.3 & 2.58 & 0.97 \\
\hline
\end{tabular}

\subsection{Chitosan hydrogel characteristics}

The chitosan biopolymer used in this study was purchased from Sigma-Aldrich (Merck) company. The material is of high molecular weight with the molecular formula C6H11NO4. The viscosity of chitosan is directly related to its molecular weight. Increasing the molecular weight and concentration of chitosan, could effectively improves its properties [23]. Acetic acid was used to make chitosan hydrogel because chitosan is insoluble in water.

To prepare the chitosan solution, chitosan in different percentages of $0.08,0.16,0.24$ and 0.32 relative to the dry weight of the soil was dissolved in water and acetic acid. To find out the amount of acetic acid required, after mixing water and chitosan powder, we added the $0.01 \mathrm{ml}$ of acetic acid by micropipette and it was mixed by stirrer for 5 minutes at each step, Figure 1. But due to the very low weight of chitosan, we considered water and chitosan powder 10 times larger for each sample.

\subsection{Specimen preparation and mechanical characterization}

The amount of soil used for each uniaxial sample was calculated based on relative density, which was assumed to be $35 \%(\mathrm{Dr}=35 \%)$. In this state the sand is very loose, and the effect of biopolymer in this state is less than that of compact [24].

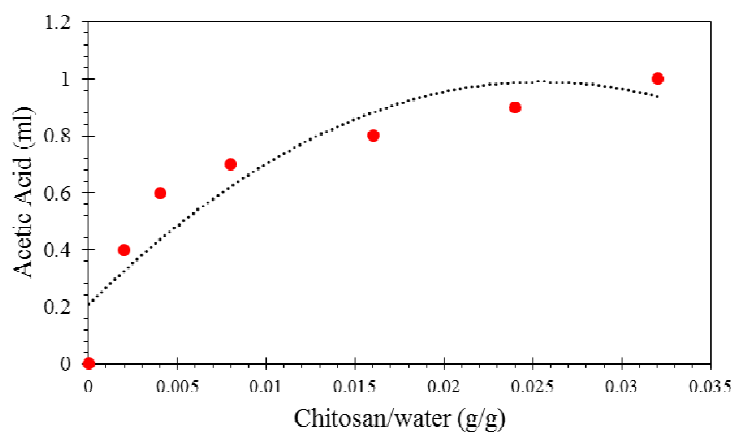

Fig. 1. Preparation of the different concentrations of the chitosan admixtures.

The samples were made at $10 \%$ moisture content and water content was calculated as a percentage of soil dry weight. After preparation of the solution the soil was manually mixed with chitosan solution for $5 \mathrm{~min}$. Samples were cured at three different conditions: dry condition (DC), wet condition (WC) and saturated in water condition (SWC) and tested after 7, 14 and 28 days treatment. At DC condition, specimens were cured at $60{ }^{\circ} \mathrm{C}$ until the testing date; At SWC condition, samples were cured in DC condition followed by 48 hours in water. At WC condition, the samples were stored in a humid chamber $(\sim 80 \pm 2 \%)$ at ambient temperature $\left(\sim 25 \pm 2{ }^{\circ} \mathrm{C}\right)$. After the course of curing time UCS testing was done.

Unconfined uniaxial compressive testing was performed with digital equipment (SH-300) on cylindrical specimens of D $38 \mathrm{~mm} \times \mathrm{H} 80 \mathrm{~mm}$. The axial strain rate was set to $0.5 \mathrm{~mm} / \mathrm{min}$. All samples were statically compacted in three layers. Each experiment was repeated three times to ensure the results of the experiments.

For permeability testing, the mold dimensions were D $70 \mathrm{~mm} \times \mathrm{H} 140 \mathrm{~mm}$. Samples were treated at OC condition and the test was performed on the samples after 5 days cuing. Hydraulic conductivity coefficient was measured by falling head permeability test.

\section{Results and analysis}

\subsection{Unconfined compressive strength (UCS) of chitosan-treated sand specimens}

The results of all the curing states at different concentrations consist of $0.08 \%, 0.16 \%, 0.24 \%$ and $0.32 \%$ of chitosan at three different days 7,14 and 28 are shown in Figure 2. In the Figure $2 \mathrm{a}$, changes in compressive strength of the specimens are observed with changes in the concentration of chitosan at different times. As can be seen, the compressive strength increases with increasing chitosan concentration but decreases with increasing time. To understand the effect of the curing conditions, we placed the samples in a humid chamber at ambient temperature, the results of which are shown in Figure $2 \mathrm{~b}$. The increase in soil 
strength in this state is not noticeable. And after reaching a certain strength, the incremental process stops and this strength stays constant over time.

The Figure 2c shows mechanical strength of specimens cured at SWC condition. This state was carried out to compare the effect of water between during treatment and after dry curing. In this state, the strength changes are similar to the DC state. In all states the strength reaches a constant value over time and becomes constant.
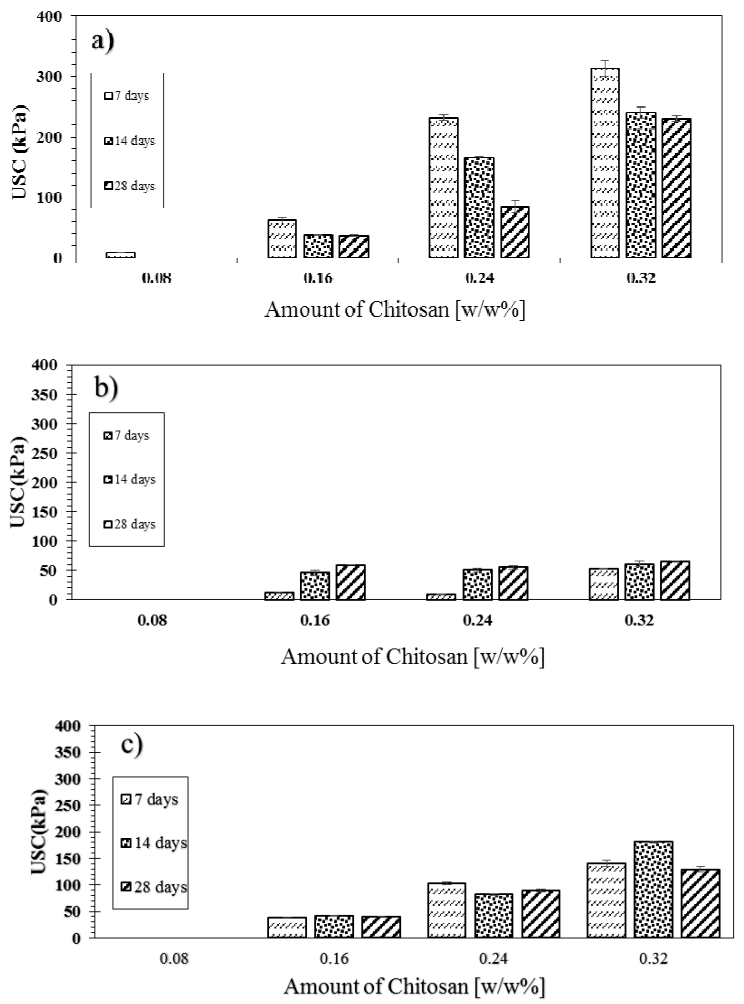

Fig. 2. Results of compressive strength tests at a) DC state. b) WC state. c) SWC state.

\subsection{Moisture content of soil specimens}

To calculate the moisture content of the specimens, samples were placed at $100^{\circ} \mathrm{C}$ for 48 hours. The results are shown in Figure 3. Moisture content is a very important factor in soil strength development in using biopolymer $[3,25]$. Therefore, to investigate this factor, the moisture content at different curing conditions was measured.

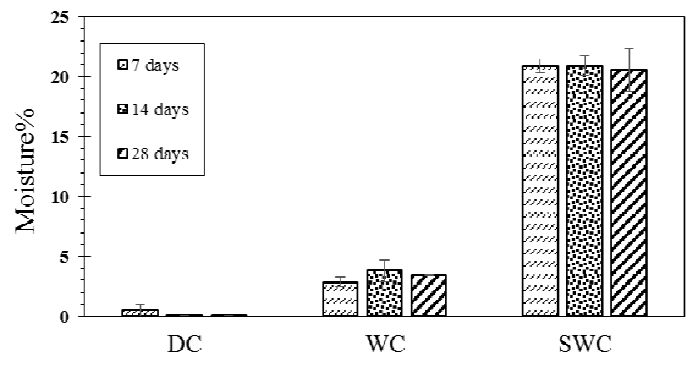

Fig. 3. Moisture content of DC, WC and SWC cured soil specimens.

\subsection{Contact angle test}

Samples were prepared for this test in two methods. In the first method, soil and chitosan were mixed with similar percentages of compressive tests. In the second method, the samples were coated with chitosan hydrogel. Sessile drop method was used to measure chitosan's ability to water impermeability of samples. In this test method contact angle is defined as the angle formed by the liquid and the surface of the material. The sample is impermeable if the angle is greater than 90 but the sample is permeable if the angle is less than 90. In this study, samples that were mixed with chitosan solution kept a drop of water for a short time and no drops were formed on their surface. This was similar in all percentages of chitosan. However, in the samples that used chitosan as a coating, water drops remained on the sample at an angle of $91^{\circ}$ in $0.24 \%$ [Chitosan/soil \%] and $124^{\circ}$ in $0.32 \%$ [Chitosan/soil \%] and this drop was still on the samples after a long time (3 hours), Figure 4. This indicates that the samples are hydrophobic and chitosan can be used as a material to reduce permeability. In chitosan mixed samples, due to the large space between the particles, sand grains only covered with chitosan hydrogel, so the water drop penetrates into the samples. Probably if the density of the samples and the amount of chitosan increased, the water would not penetrate into these samples. Similar results were reported by other researchers.

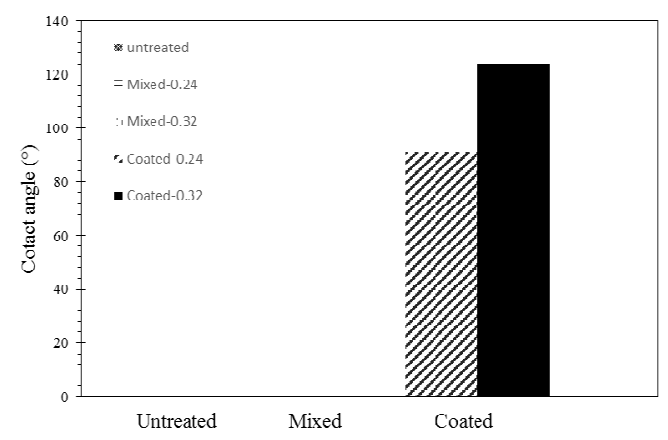

Fig. 4. Comparison of $0.24 \%[\mathrm{w} / \mathrm{w} \%]$ and $0.32 \%[\mathrm{w} / \mathrm{w}]$ contact angle test results.

\subsection{Hydraulic conductivity}

The permeability test results show that chitosan significantly reduces permeability. As shown in Figure 8 , the hydraulic conductivity coefficient decreased from $1.6 \times 10^{-6}$ in untreated sandy soil to $7.3 \times 10^{-7}$ in Chitosan $0.24 \%$ [w/w\%] and $5.7 \times 10^{-7}$ in Chitosan $0.32 \%$ [w/w\%], Figure 5. According to contact angle testing these results were expected. As the concentration of chitosan increases, the permeability decreases. 


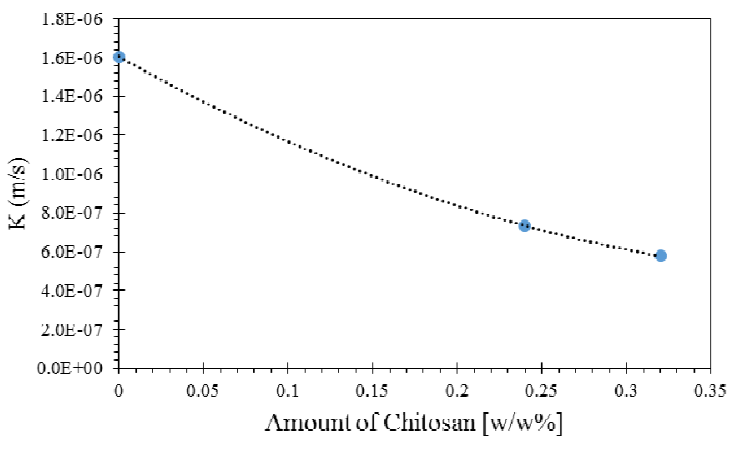

Fig. 5. Results of hydraulic conductivity test.

\section{Discussion}

The results of the compressive strength tests are shown in the Figure 2. The results show that to improve soil strength, the sample must be dried so that chitosan can improve soil cohesion. Because it is observed in the case that the treatment was performed in a humid environment, so the strength of the specimens did not increase significantly. As shown in Figure 3, the moisture content of the SWC state is much greater than that of the WC state, but SWC state strength is greater than the WC state. This indicates that the presence of moisture at the time of curing processing is an effective factor in strength development, but after drying, the effect of moisture and water is much less. One reason for this is that the improved samples with chitosan are hydrophobic. Some researchers have reported that over time, the strength of clay samples come back to untreated state. But this trend was not observed in this study. This may be due to differences in the type of chitosan material and the different methods of chitosan synthesis, as well as differences in soil type. As mentioned, molecular weight and degree of acetylation are two very important factors in chitosan property [15, 26].

The hypothesis of chitosan-clay micro behaviour is associated to cationic characteristics of chitosan which provides an electrical interaction between the biopolymer and the diffuse double layer of clay minerals (the charged surface and the distributed charge in the adjacent phase) that governs the inter-particle behaviour of the treated clay. While, electro-static and hydrogen bonding phenomena cannot be expected for sand particles, because sand particles carry no electrical charges. Thus, the basic properties of chitosan are preserved, including water insolubility and the strength of chitosan-coarse soil mixture strongly depends on the strength of chitosan gels.

In cohesionless soils, porosity is a very dominant factor in soil strength, and it is highly effective when the porosity is lower. Continuous biopolymer film bridges formation is mostly affective to strengthening effect rather than bridge length, bonding direction and biopolymer concentration. If the samples became more compact, higher strength would probably be obtained, that's because of the high effective surface area and low distance between grains in compacted soils.

\section{Conclusions}

Chitosan is an effective soil stabilizer, which is effective in long-term sandy soils and in short-term clay soils. This is due to the formation of electrostatic bonds between the clay and chitosan particles, which does not occur in the sand because the sand surface has a low electric charge. This causes the chitosan biopolymer property in the sandy soil to remain constant over time.

Soil moisture content is a very important factor in enhancement if soil mechanical and hydraulic properties by chitosan, which has a much greater effect on curing time. The specimens treated at DC state had the highest strength to the WC and SWC states. At SWC state, submerged specimens show good strength.

Contact angle and permeability tests show that chitosan can reduce the permeability of sand so that the higher the concentration of chitosan, the lower the permeability of sand.

If chitosan is used to counteract water penetration, chitosan as a coating is much more effective than mixing it with materials.

\section{Acknowledgements}

The project has received funding from the European Union's Horizon 2020 research and innovation programme under the Marie Sklodowska-Curie grant agreement No 778120.

\section{References}

1. B. McGuire, Waking the giant: How a changing climate triggers earthquakes, tsunamis, and volcanoes, Oxford University Press, (2013).

2. M.S. Imbabi, C. Carrigan, S.J.I.J.O.S.B.E. McKenna, Trends and developments in green cement and concrete technology, 1 (2012) 194-216.

3. N. Hataf, P. Ghadir, N.J.J.O.C.P. Ranjbar, Investigation of soil stabilization using chitosan biopolymer, 170 (2018) 1493-1500.

4. W. Pu, C. Shen, B. Wei, Y. Yang, Y. Li, A comprehensive review of polysaccharide biopolymers for enhanced oil recovery (EOR) from flask to field, Journal of Industrial and Engineering Chemistry, 61 (2018) 1-11.

5. A. Mosallanejad, H. Taghvaei, S.M. Mirsoleimaniazizi, A. Mohammadi, M.R. Rahimpour, Plasma upgrading of 4methylanisole: A novel approach for hydrodeoxygenation of bio oil without using a hydrogen source, Chemical Engineering Research and Design, 121 (2017) 113-124.

6. S. Boonlertnirun, C. Boonraung, R.J.J.O.M. Suvanasara, materials, minerals, Application of chitosan in rice production, 18 (2017).

7. A. Uthairatanakij, J. Teixeira da Silva, K.J.O.S. Obsuwan, Biotechnology, Chitosan for improving orchid production and quality, 1 (2007) 1-5.

8. H.R. Khatami, B.C.J.J.O.G. O'Kelly, G. Engineering, Improving mechanical properties of sand using biopolymers, 139 (2012) 1402-1406. 
9. I. Chang, G.C.J.A.G. Cho, Shear strength behavior and parameters of microbial gellan gum-treated soils: from sand to clay, 14 (2019) 361-375.

10. I. Chang, J. Im, A.K. Prasidhi, G.C.J.C. Cho, B. Materials, Effects of Xanthan gum biopolymer on soil strengthening, 74 (2015) 65-72.

11. N. Latifi, S. Horpibulsuk, C.L. Meehan, M.Z. Abd Majid, M.M. Tahir, E.T.J.J.o.M.i.C.E. Mohamad, Improvement of problematic soils with biopolymeran environmentally friendly soil stabilizer, 29 (2016) 04016204.

12. I. Chang, A.K. Prasidhi, J. Im, G.C.J.C. Cho, B. Materials, Soil strengthening using thermo-gelation biopolymers, 77 (2015) 430-438.

13. I. Chang, J. Im, G.C.J.C.G.J. Cho, Geotechnical engineering behaviors of gellan gum biopolymer treated sand, 53 (2016) 1658-1670.

14. I. Chang, J. Im, S.-W. Lee, G.C.J.C. Cho, B. Materials, Strength durability of gellan gum biopolymer-treated Korean sand with cyclic wetting and drying, 143 (2017) 210-221.

15. I. Younes, M.J.M.D. Rinaudo, Chitin and chitosan preparation from marine sources. Structure, properties and applications, 13 (2015) 1133-1174.

16. D.J.F.T. Knorr, Recovery and utilization of chitin and chitosan in food processing waste management, (1991).

17. C. Escudero-Oñate, E.J.C.C.M.F.I.S. MartínezFrancés, Technology, A Review of Chitosan-Based Materials for the Removal of Organic Pollution from Water and Bioaugmentation, (2018) 71.

18. P.O. Boamah, Y. Huang, M. Hua, Q. Zhang, J. Wu, J. Onumah, L.K. Sam-Amoah, P.O.J.E. Boamah, E. Safety, Sorption of heavy metal ions onto carboxylate chitosan derivatives-a mini-review, 116 (2015) 113-120.

19. N. Saberi, M. Aghababaei, M. Ostovar, H. Mehrnahad, Simultaneous removal of polycyclic aromatic hydrocarbon and heavy metals from an artificial clayey soil by enhanced electrokinetic method, Journal of Environmental Management, 217 (2018) 897-905.

20. E. Kavazanjian Jr, E. Iglesias, I. Karatas, Biopolymer soil stabilization for wind erosion control, in: Proc. 17th Int. Conf. Soil Mech. Geotech. Engng, Alexandria, (2009) 881-884.

21. A. Alsanad, Novel biopolymer treatment for wind induced soil erosion, Arizona State University, (2011).

22. R. Aguilar, J. Nakamatsu, E. Ramírez, M. Elgegren, J. Ayarza, S. Kim, M.A. Pando, L.J.C. Ortega-SanMartin, B. Materials, The potential use of chitosan as a biopolymer additive for enhanced mechanical properties and water resistance of earthen construction, 114 (2016) 625-637.

23. K. Tai, M. Rappolt, L. Mao, Y. Gao, X. Li, F.J.F.H. Yuan, The stabilization and release performances of curcumin-loaded liposomes coated by high and low molecular weight chitosan, (2019) 105355.

24. S. Lee, I. Chang, Microscopic investigation of interparticle-interaction between sand particles and biopolymer, (2019).

25. P. Ghadir, N. Ranjbar, Clayey soil stabilization using geopolymer and Portland cement, Construction and Building Materials, 188 (2018) 361-371.

26. Y. Yuan, B.M. Chesnutt, W.O. Haggard, J.D.J.M. Bumgardner, Deacetylation of chitosan: material characterization and in vitro evaluation via albumin adsorption and pre-osteoblastic cell cultures, 4 (2011) 1399-1416. 